\title{
Circadian rhythms influence surgical outcomes
}

myocardial
tolerance to
ischaemia-
reperfusion
differs during
the day

The outcomes of cardiac surgery could be better if the procedure is performed in the afternoon rather than in the morning. This effect seems to be transcriptionally controlled by the circadian clock, can be pharmacologically targeted, and might be relevant when scheduling high-risk patients for operations.

David Montaigne, Bart Staels, and colleagues studied the incidence of major adverse cardiovascular events (MACE; cardiovascular death, myocardial infarction, and admission to hospital for acute heart failure) in a prospective, observational cohort of 720 patients with severe aortic stenosis and preserved left ventricular ejection fraction (>50\%) who were undergoing aortic valve replacement at Lille University Hospital, France. One of four senior surgeons performed all operations, and each surgeon operated twice each working day; the same surgical team did both morning and afternoon surgery on the same day. Propensity score matching was used to generate two well-matched populations of 298 patients. After follow-up (median 500 days), the frequency of MACE was lower with afternoon surgery than with morning surgery ( $9 \%$ versus $18 \%$; HR $0.50,95 \% \mathrm{Cl} 0.32-0.77, P<0.0021)$. Both perioperative myocardial infarction and acute heart failure were significantly reduced with afternoon versus morning surgery.

Next, the investigators randomly assigned 88 patients to undergo isolated aortic valve replacement in either the morning or the afternoon. Perioperative myocardial injury, assessed by measuring cardiac troponin T release, was significantly lower in the afternoon group than in the morning group $(179 \mathrm{ng} / \mathrm{l}$ versus $225 \mathrm{ng} / \mathrm{l}$; estimated ratio of geometric means $0.79,95 \% \mathrm{Cl} 0.68-0.93, P=0.0045$ ).

Next, the researchers subjected human myocardial samples to hypoxiareoxygenation conditions ex vivo. Contraction recovery of the biopsy samples was significantly better in atrial myocardium collected from patients undergoing afternoon surgery than in those from patients undergoing morning surgery. A transcriptomic analysis of these biopsy samples showed that the expression of 287 genes was regulated by the time of day. Levels of the nuclear receptor transcriptional repressor REV-ERB $\alpha$ (also known as nuclear receptor subfamily 1 group D member 1; NR1D1) were low in the afternoon, resulting in high expression of its target gene, ARNTL (also known as BMAL1).

Inhibition of REV-ERBa in mouse hearts, either by knocking out the Nr1d1 gene or with a pharmacological antagonist (SR8278), conferred hypoxia-reoxygenation tolerance through an increase in the levels of the ischaemia-reperfusion modulator cyclin-dependent kinase inhibitor 1 (also known as p21).

"We demonstrated for the first time in humans that the myocardial tolerance to ischaemia-reperfusion differs during the day," conclude Montaigne and Staels. "This biorhythm is clinically meaningful and can be targeted as a cardioprotective strategy." They note that their findings are consistent with established concepts of chronobiology and chronotherapy. "Time of day must be part of the design and interpretation of both bench-side and clinical studies regarding the impact of ischaemia-reperfusion injury," they remark.

Gerd Heusch from the University of Essen, Germany, and who was not involved in the study, notes the relevance of the work, given that the 2017 Nobel Prize for Physiology or Medicine has just been awarded for studies on circadian rhythm. "A pragmatic conclusion from this elegant study is to perform high-risk cardiovascular surgery in the afternoon rather than the morning," suggests Heusch. "Conceptually, the gene encoding REV-ERB $\alpha$ and its resulting signal transduction could be an interesting target for pharmacological development of adjunct cardioprotection."

Gregory B. Lim

ORIGINAL ARTICLE Montaigne, D. et al. Daytime variation of perioperative myocardial injury in cardiac surgery and its prevention by Rev-Erba antagonism: a single-centre propensity-matched cohort study and a randomised study. Lancet http://dx.doi.org/10.1016/S0140-6736(17)32132-3 (2017) FURTHER READING Hausenloy, D. J. \& Yellon, D. M. Ischaemic conditioning and reperfusion injury. Nat. Rev. Cardiol. 13, 193-209 (2016) 